\title{
SLOWNESS SURFACES AND ENERGY FOCUSING PATTERNS OF AUXETIC CUBIC MEDIA
}

\author{
T. PASZKIEWICZ', M. PRUCHNIK² AND S. WOLSKI ${ }^{1}$ \\ ${ }^{1}$ Chair of Physics, Rzeszów University of Technology \\ W. Pola 2, PL-35-959 Rzeszów, Poland \\ ${ }^{2}$ Institute of Theoretical Physics University of Wrockaw \\ Pl. Maxa Borna 9, PL-50-204 Wrockaw, Poland
}

(Rec. 29 September 2004)

\begin{abstract}
We study properties of slowness surfaces and energy focusing patterns of cubic elastic media. We restricted ourselves to the region of the stability triangle where Poisson's ratio $\sigma_{P}$ of the specimen stretched in the [001] direction and measured for [100] is negative, i.e. we consider all cubic auxetic materials. We study properties of surfaces and energy focusing patterns for all elastic auxetic media characterized by $\sigma_{P}=-1 / 3$.
\end{abstract}

\section{INTRODUCTION}

Recently there is considerable interest in auxetics - elastic materials with negative Poisson's ratio (cf. [1-3]). Generally the calculation of Poisson's ratio is complicated for directions oblique to the crystal axes [4]. Therefore, in our previous papers [5, 6] we restricted ourselves to Poisson's ratio for [001] stretch measured for [100] lateral direction and established the region of the stability triangle (ST) (cf. [6]) where the mentioned Poisson's ratio is negative. We shall underline that the characteristics of particular region of auxeticity depends on the choice of the stretch direction $\mathbf{n}$ and the direction $\mathbf{m}$ of measurement [7]. To indicate this dependence we shall use notation $\mathbf{n m}$-auxeticity region of ST.

The present paper is devoted to the study of geometrical properties of slowness surfaces of long wavelength acoustic phonons [8,9] in the [001][100]-auxeticity region (for simplicity we shall use a short notation $z x$-Poisson's ratio and $z x$-auxeticity region). We also obtained the energy focusing patterns $[8,9]$ which are images of these surfaces under the mapping induced by phonon focusing, which is mathematically known as the Gauss maps (cf. [10]). These maps are obtained in time-of-flight experiments with ballistic phonon beams [8, 9].

\section{CHOICE OF ELASTICITY PARAMETERS}

\subsection{Partition of the stability triangle}

Within the framework of theory of elastic media, in a chosen Cartesian coordinate system, the phase $\mathrm{c}$ and group velocities $\mathbf{v}$, as well as polarization vectors e of long wavelength acous- 
tic phonons depend on parameters characterizing elastic properties of the medium. The most commonly used is the set of elastic constants $\left\{C_{i j}: i, j=1,2,3\right\}$ and the mass density $\rho$. However, following Every [11], in our previous papers [12, 13] for cubic crystals we used a linear combination of $C_{11}$ and $C_{44}$

$$
s_{1}=\left(C_{11}+2 C_{44}\right)
$$

and two dimensionless parameters $s_{2}, s_{3}$

$$
s_{2}=\frac{\left(C_{11}-C_{44}\right)}{s_{1}}, \quad s_{3}=\frac{\left(C_{11}-C_{12}-2 C_{44}\right)}{s_{1}} .
$$

Notice that the triple $\left(s_{1}, s_{2}, s_{3}\right)$ is equivalent to the set $\left(C_{11}, C_{12}, C_{44}\right)$ of elastic constants. Since the parameter $s_{3}$ vanishes for isotropic media, we shall call it the elastic anisotropy parameter. The parameter $s_{1}$ is a scaling parameter. The phase and group velocities are proportional to it. The Poisson ratio does not depend on $s_{1}$.

We shall remind that elastic properties of all media belonging to the cubic crystallographic system, i.e. to the crystallographic classes $23(T), \overline{4} 3 m\left(T_{d}\right), m 3\left(T_{h}\right), 432(O)$ and $m 3 m\left(O_{h}\right)$, are described by the same set of parameters of elasticity.

The condition of mechanical stability imposes certain restrictions on values of the parameters $s_{1}, s_{2}$ and $s_{3}$. Namely, $s_{1}$ should be positive and

$$
\left(1+2 s_{2}\right)>\left|4 s_{2}-3 s_{3}-1\right|, \quad\left(10 s_{2}-6 s_{3}\right)>1, \quad s_{2}<1 .
$$

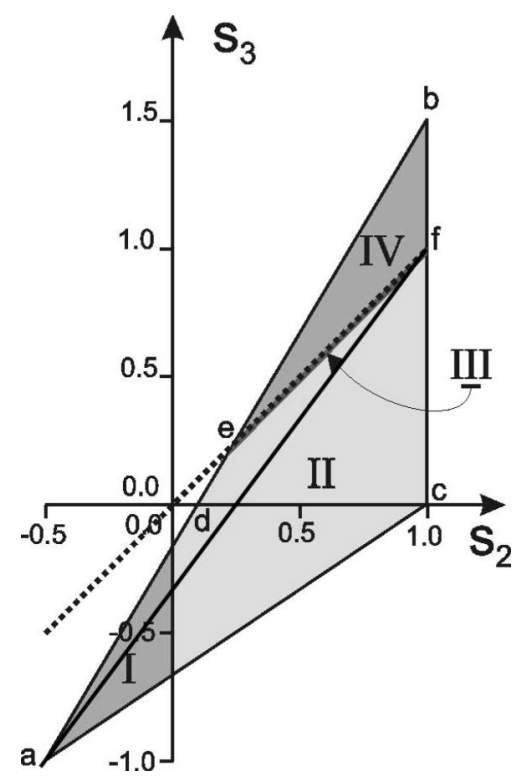

Fig. 1. Various regions of the stability triangle $a b c$. The polarization anomalies line (PAL) $s_{2}=s_{3}$ (i.e. $C_{12}=-C_{44}$ ) is indicated (the dotted line). The line $s_{2}=0$ is called the vertical polarization anomalies line (VPAL) [5]. On the $a f$-line the $z x$-Poisson ratio attains the value 0 
These inequalities mean that $s_{2}$ and $s_{3}$ can only vary inside the stability triangle (abc triangle in Fig. 1) bounded by $s_{3}=\frac{5}{3} s_{2}-\frac{1}{6}$ ( $a b$-side), $s_{3}=\frac{2}{3} s_{2}-\frac{2}{3}$ ( $a c$-side) and $s_{2}=1$ ( $b c$-side) lines.

As we shown [5], the stability triangle $a b c$, can be divided into four separate regions in which corresponding crystalline media exhibit different acoustic properties. These regions are shown in Fig. 1. The region I is lying left to the $s_{3}$ axis, i.e. for it $s_{2}<0$. We shall call the line $s_{2}=0$ the vertical polarization anomalies line (VPAL). The region II is located between VPAL and the line which we call the polarization anomalies line (PAL), which lies slightly below the acoustic anomalies line (AAL), i.e. $s_{3}=s_{2}$ line. The PAL can be found only numerically, and not necessarily is straight. The region III is placed between PAL and AAL, while region IV is a part of the stability triangle lying above AAL.

\section{2. $z x$-Poisson's ratio}

Consider the dependence of the $z x$-Poisson ratio $\sigma_{P}$ on parameters of elasticity

$$
\sigma_{p}=\frac{C_{12}}{C_{11}+C_{12}}=\frac{\left(4 s_{2}-3 s_{3}-1\right)}{3\left(2 s_{2}-s_{3}\right)} \text {. }
$$

The $z x$-Poisson ratio $\sigma_{P}$ vanishes for $C_{12}=0$, i.e. on the section $\overline{a f}$ of $s_{3}=\frac{1}{3}\left(4 s_{2}-1\right)$ line. Above this line $\sigma_{P}$ is negative while below it is positive. According [14] in the case of cubic intermediate valent compounds the vanishing $\sigma_{\mathrm{P}}$ indicates the semiconductor-metal transition. Plot of $\sigma_{P}$ is presented in Fig. 2.

We shall underline that the region of auxeticity (cf. also Fig. 3) forms a finite part of the stability triangle, i.e. defines a continuous set of finite measure of cubic auxetic compounds. Hence, one may expect that auxeticity could be a common phenomenon.

Fig. 2. Dependence of the $z x$-Poisson ratio $\sigma_{p}$ on $s_{2}$ and $s_{3}$. On $\overline{a^{\prime} f^{\prime}}$ the Poisson ratio vanishes. Left to $\overline{a f}$ (the projection of $\overline{a^{\prime} f^{\prime}}$ onto the plane $\left.\sigma_{p}=-1.0\right)$, hence $\sigma_{p}<0$ also on $\overline{a b}$. Right to $\overline{a f} \sigma_{p}>0$

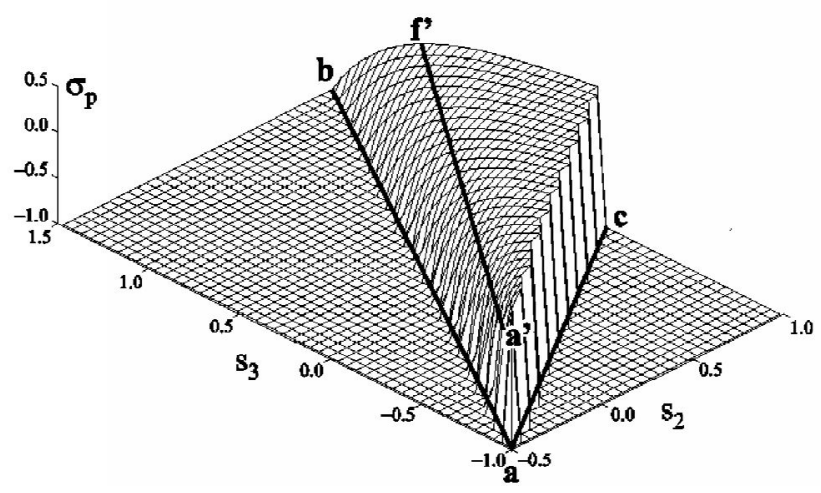

In Sect. 3.3 we shall study properties of slowness surfaces in $z x$-region of auxeticity along a line of constant value of $\sigma_{P}$.

From Eq. 4 it follows that the parameters $s_{2}$ and $s_{3}$ are related by a linear relation 


$$
s_{3}=\frac{2\left(2-3 \sigma_{P}\right)}{3\left(1-\sigma_{P}\right)} s_{2}-\frac{1}{3\left(1-\sigma_{P}\right)} .
$$

From Eq. 5 it follows that to the borders of auxeticity region correspond to $\sigma_{P}=0$ (the line af in Fig. 1) and $\sigma_{P}=-1$ (the $a b$-side of the stability triangle (cf. Fig. 1)).

\section{PROPERTIES OF LONG-WAVELENGTH ACOUSTIC PHONONS}

Weakly disturbed states of crystalline lattices can be described in terms of phonons. Here we consider only long wavelength acoustic phonons (LAPs) - fast $(j=0)$, slow $(j=1)$ and medium $(j=2)$ [11]. As a rule the fast mode is quasilongitudinal and the remaining modes are quasitransverse. However, there exists regions on ST and directions for which $j=0$ mode is quasitransverse and at least one of the remaining modes is quasilongitudinal [5] (cf. also Sect. 3.4).

\subsection{Phase and group velocities of long-wavelength phonons}

A phonon carries the energy $\varepsilon=\hbar \omega$ and quasimomentum $\mathbf{p}=\hbar \mathbf{q}$. The energy $\varepsilon$ and, of course, the frequency $\omega$, depend on the wave vector $\mathbf{q}$ and on the index $j$. The dispersion law of LAPs is linear in the magnitude $q$ of the wave vector

$$
\omega(\mathbf{q}, j)=c(\mathbf{q}, j) q \quad(\mathrm{j}=0,1,2) .
$$

The coefficient $c$, called the phase (sound) velocity, depends only on the polarization $j$ and the direction of the wave vector $\hat{\mathbf{q}}$ (or on the angles $\theta_{q}$ and $\phi_{q}$ ). We shall use a brief notation $Q \equiv(\mathbf{q}, j)$ and $\hat{Q} \equiv(\hat{\mathbf{q}}, j)$.

For long wavelength acoustic phonons the group velocity also depends only on $\hat{Q}$. The group velocity vector

$$
v_{\alpha}(\mathbf{q}, j)=\frac{\partial \varepsilon(\mathbf{p}, j)}{\partial p_{\alpha}}=\frac{\partial \omega(\mathbf{q}, j)}{\partial q_{\alpha}},
$$

has the direction of the maximum rate of the frequency change and is perpendicular to a $j$-th sheet of surface of constant frequency ( $\omega$ - surface), which is defined as

$$
\omega(\mathbf{q}, j)=\omega_{0} \quad\left(\omega_{0}=\text { const }, \quad j=0,1,2\right) .
$$

\subsection{Local geometrical characteristics of slowness surfaces}

Having the phase velocity $c(\hat{Q})$ one can introduce the slowness $s(\hat{Q})$, namely

$$
s(\hat{Q})=c^{-1}(\hat{Q})
$$

The slowness $s(\hat{Q})$ of LAPs depends on $\hat{Q}$ and on parameters of elasticity. 
For each $j$ the polar plot of the slowness gives a slowness surface (SIS). For LAPs the equation of a slowness surface is equivalent to

$$
\mathbf{q}_{j}=s(\hat{Q}) \omega_{0} \hat{\mathbf{q}} \quad(j=0,1,2) .
$$

For each $j$ the constant frequency surface, or constant energy surface, is similar to the corresponding slowness surface. Thus, an $\omega$-surface of LAPs of $j$-th branch has the same shape as the corresponding slowness surface. Each slowness surface has the symmetry of the considered crystalline medium and generally a nonspherical shape. Similarly as the Fermi surface of metals, the slowness surfaces plays a distinguished role in transport phenomena in gases of LAPs. Further we shall term $j=0$ SIS - the inner sheet, $j=1$ SIS - the outer sheet, and $j=2$ - the middle sheet.

Assume that long wavelength phonons are radiated in form of very short pulses by a point source located at the origin of a Cartesian coordinate system. An energy sensitive detector (a bolometer) is placed at the point $\mathbf{r}=\left(r, \theta_{r}, r\right)$ lying in a plane with a normal $\hat{\mathbf{n}}$. A phonon with the wave vector $\mathbf{q}=\left(q, \theta_{q},{ }_{q}\right)$ falls onto the detector if its group velocity direction $\hat{\mathbf{v}}\left(\theta_{v}^{(j)}, \phi_{v}^{(j)}\right)$ coincides with the direction of the radius vector $\mathbf{r}$ of the detector. Generally, due to the complicated nonspherical shape of surfaces of constant energy, there is more than one solution to the vectorial equation

$$
\hat{\mathbf{r}}\left(\theta_{r}, \phi_{r}\right)=\hat{\mathbf{v}}_{j}\left(\theta_{j}, \phi_{v}\right)
$$

or to the two equations for angles

$$
\theta_{r}=\theta_{v}^{(j)}\left(\theta_{q}, \phi_{q}\right), \quad \phi_{r}=\phi_{v}^{(j)}\left(\theta_{q}, \phi_{q}\right) \quad(j=0,1,2)
$$

Denote them by $\theta_{q j}^{(i)}\left(\theta_{r}, \phi_{r}\right), \phi_{q j}^{(i)}\left(\theta_{r}, \phi_{r}\right),\left(i=1,2, \ldots, n_{j}\right)$. For an arbitrary function $F_{j}$ of $\hat{\mathbf{r}} \equiv\left(\theta_{r}, \phi_{r}\right)$ we introduce the notation

$$
F_{j}^{(i)}\left(\theta_{r}, \phi_{r}\right) \equiv F_{j}\left[\theta_{q j}^{(i)}\left(\theta_{r}, \phi_{r}\right), \phi_{q j}^{(i)}\left(\theta_{r}, \phi_{r}\right)\right] .
$$

The total density of energy $e_{n}(\mathbf{r})$ of LAPs falling on a small region around a point $\mathbf{r}$ of the surface with the normal $\hat{\mathbf{n}}$ can be expressed by the focusing (enhancement factor) [12]

$$
e_{n}(\mathbf{r})=\frac{(\hat{\mathbf{n}} \cdot \hat{\mathbf{r}}) \hbar \omega_{0}}{2 \pi r^{2}} \frac{1}{3} \sum_{j=0}^{2} \sum_{i=1}^{n_{j}} \mathrm{~A}_{j}^{(i)}\left(\theta_{r}, \phi_{r}\right) .
$$

The focusing factor is related to the local geometric characteristics of the suitable slowness-surface at the point of intersection of this surface with the vector q. These local characteristics are the length of the vector $\mathbf{q}$, the angle between $\mathbf{q}$ and $\boldsymbol{v}_{j}$ and the Gaussian curvature $\Gamma_{j}$ of the $j$-th sheet of slowness surface, and

$$
\mathrm{A}_{j}\left(\theta_{j}, \phi_{q}\right)=\left|\left(\hat{\mathbf{v}}_{j} \cdot \hat{\mathbf{q}}\right)\right|\left[\Gamma_{j}(\hat{\mathbf{q}}) q^{2}\right]^{-1} .
$$


On parabolic lines and at flattening points (e.g. the points of crossing of parabolic lines) of an slowness surface the Gaussian curvature vanishes, hence at such points the densities of energy and of quasimomentum are singular.

\subsection{Slowness surfaces in $z x$-auxetic region}

Assume that $\sigma_{P}=-1 / 3$. To this value of the $z x$-Poisson's ratio it corresponds the line

$$
s_{3}=\frac{3}{2} s_{2}-\frac{1}{4} .
$$

The $\sigma_{P}=-1 / 3$-line defined by Eq. (16) lies in the middle of the $z x$-auxeticity region (cf. Fig. 3). We distinguished on this line seven points indicated in Fig. 3.

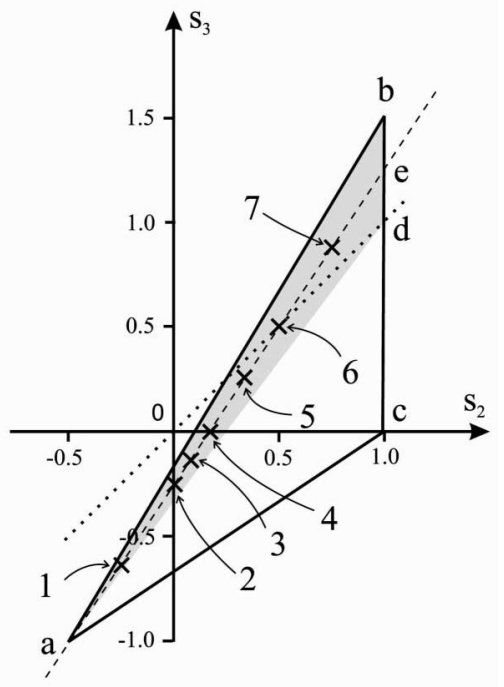

Fig. 3. Cubic crystals are mechanically stable for $s_{1}>0$ and for $s_{2}$ and $s_{3}$ belonging to the triangle $a b c$. In the shaded region $C_{12}<0$. The interval $\overline{0 c}$ (isotropy line) represents stable isotropic media. The dotted line is the polarization anomalies line. The dashed line is defined by Eq. (16). Points of the $z x$-auxeticity region for which slowness surfaces and energy focusing patterns are calculated are indicated by $\times$ sign

Notice that the line given by Eq. (16) crosses all four regions shown in Fig. 1. In our previous paper [5] we studied selected properties of SIS in all these regions of ST. Hence we can make reference to results obtained there.

In the regions II and III of the stability triangle the middle $\left(\Sigma_{2}\right)$ and the outer $\left(\Sigma_{1}\right)$ surfaces touch each other tangentially in the $\langle 100\rangle$ and conically in the $\langle 111\rangle$ directions. The inner sheet $\Sigma_{0}$ is completely separated from the remaining two sheets. In the region I the behavior of the slowness surfaces is different. For example on VPAL $\left(s_{2}=0\right) \Sigma_{0}$ makes tangential contact with the $\Sigma_{1}$ and the $\Sigma_{2}$ along $\langle 100\rangle$ directions, while $\Sigma_{1}$ and $\Sigma_{2}$ sheets touch each other at $\langle 100\rangle$ and conically in $\langle 111\rangle$ directions (cf. Fig. 4). Situation in the region IV is opposite $\Sigma_{0}$ and $\Sigma_{2}$ sheets meet conically in directions of threefold symmetry axes, while $\Sigma_{1}$ and $\Sigma_{2}$ tangentially in directions of fourfold ones.

On AAL line $\Sigma_{1}$ and $\Sigma_{2}$ contact not only along the $\langle 001\rangle$ and the $\langle 111\rangle$, but also along whole lines connecting these and equivalent points of the unit sphere. The $\Sigma_{0}$ and $\Sigma_{2}$ sheets 
contact along lines connecting the neighboring $\langle 111\rangle$. Since small changes of elasticity parameters lift these degeneracies along lines (of course leaving unchanged degeneracies along acoustic axes) they are unstable.

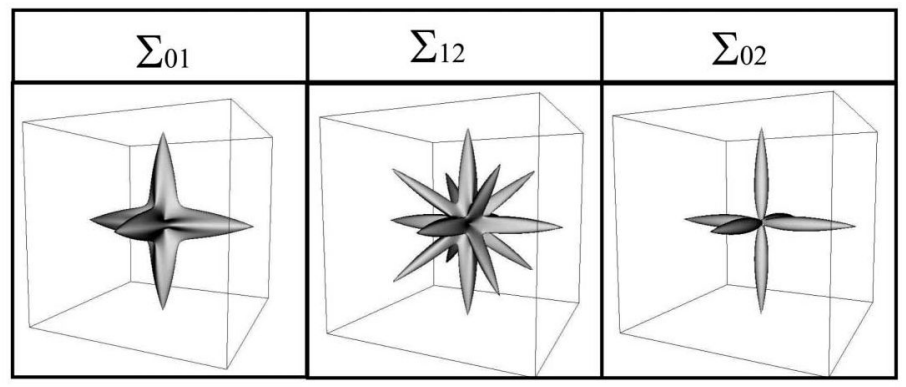

Fig. 4. Extremal points of $\Sigma_{i j}$ surfaces indicate directions in which $i$ and $j(i, j=1,2,3, i \neq j)$ sheets of SIS touches each other for point no. 2 of the $\sigma_{P}=-1 / 3$-line, for which $\mathrm{S}_{2}=0.00, \mathrm{~S}_{3}=-0.25$. Edges of cubes are directed along $\langle 001\rangle$ rangle directions

The most straightforward way of representing degenerate directions is to calculate surfaces of slownesses difference, i.e. calculate $\Delta s_{i j}(\mathbf{n})=\left|s_{i}(\mathbf{n})-s_{j}(\mathbf{n})\right|$ and vectors

$$
\Delta s_{i j}=\Delta s_{i j}(\mathbf{n}) \mathbf{n}=\left|s_{i}(\mathbf{n})-s_{j}(\mathbf{n})\right| \mathbf{n} .
$$

For given $i$ and $j$ the tips of these vectors defines a surface, which we shall call $\Delta \mathrm{s}_{i j}$-surface. Because each slowness surface consists of three sheets, for a given crystalline medium there are three different such surfaces, namely $\Delta s_{20}, \Delta s_{12}$ and $\Delta s_{10}$. From the definition of the $\Delta s_{i j}$--surfaces it is obvious that at degenerate directions these surfaces attain the value 0 .

The above approach is simple and efficient, but has two disadvantages: it is hard to precisely locate degenerate directions and it is not easy to distinguish between directions in which phase velocities are close and directions in which they are equal. To overcome this difficulty we transformed the $\Delta s_{i j}$-surfaces: the global maximum $\Delta s_{i j}^{(\max )}$ of a $\Delta s_{i j}$-surface is found, and new vectors are introduced

$$
\Delta s_{i j}^{(\mathrm{mir})}(\mathbf{n})=\left\{\frac{\left[2 \Delta s_{i j}^{(\max )}-\Delta s_{i j}(\mathbf{n})\right]}{2 \Delta s_{i j}^{(\max )}}\right\}^{5} \mathbf{n} .
$$

For each pair $(i, j)$ the tips of these vectors form a surface which we shall call $\Sigma_{i j}$-surface. In fact these new surfaces are normalized mirror images of the $\Delta s_{i j}$, where the mirrors are spheres of radii $\Delta s_{i j}^{(\max )}$. Note that for degenerate directions the $\Delta s_{i j}^{(\operatorname{mir})}$ reach the global maxima equal exactly to 1 .

We built a program which calculates and depicts $\Sigma_{i j}$-surfaces and additionally we implemented techniques which allows one to establish their polar coordinates. Results obtained with this program for the point no. 2 of the $\sigma_{P}=-1 / 3$-line are presented in Fig. 4. 
Table 1. Sheets of slowness surface for cubic elastic media for all long wavelength acoustic phonons in points nos. 1-7 of $\sigma_{p}=-1 / 3$ line. In fact various sheets differ by linear dimensions. Regions where $\Gamma<0$ are shaded. Regions with positive Gauss curvature are white. The borders of these regions indicate lines of parabolic points $(\Gamma=0)$

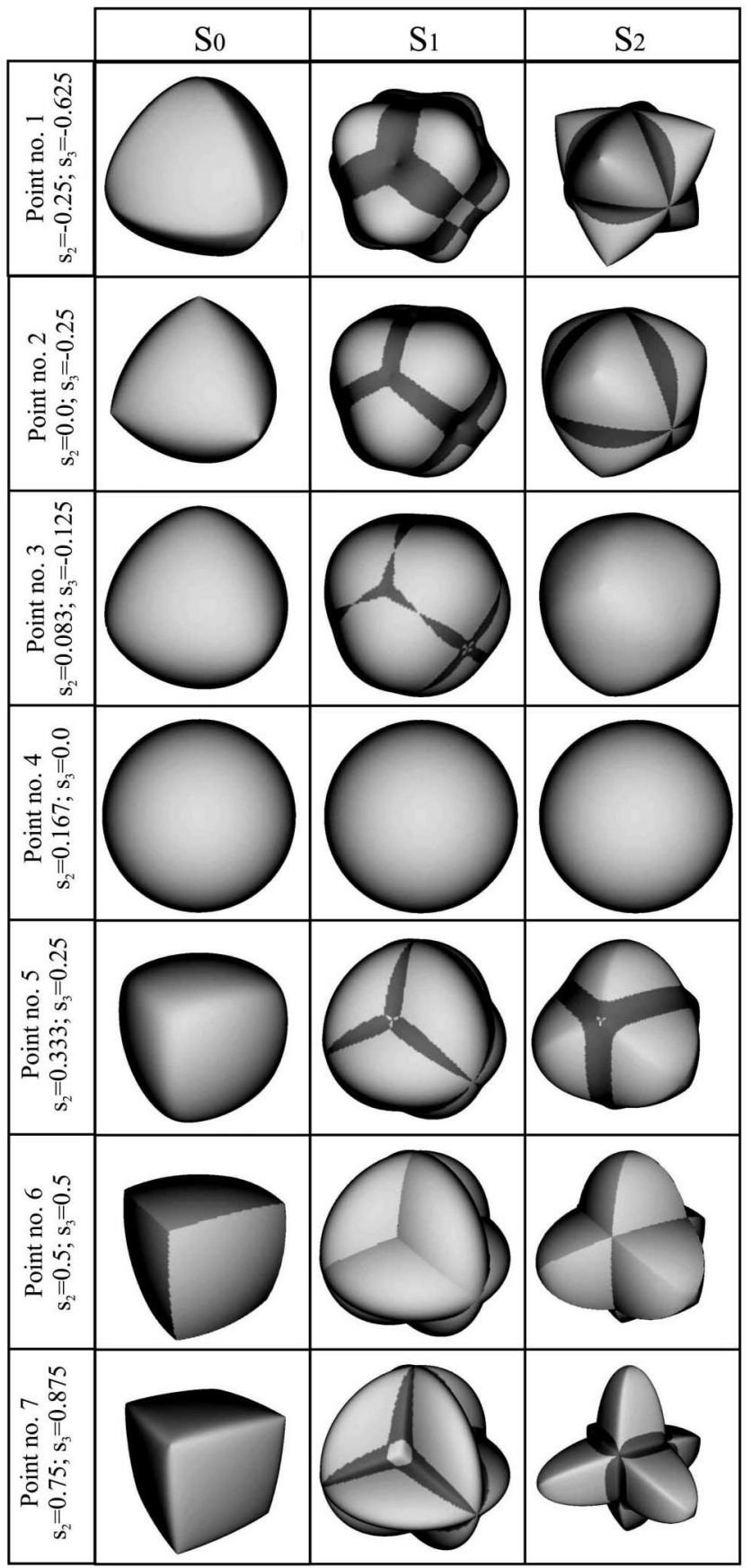


Results of more systematic calculations of the slowness surfaces are presented in Table 1 . The curvature of regions of slowness surfaces are indicated: shaded regions have negative, whereas white regions - positive Gauss curvature. Therefore, on lines separating them $\Gamma=0$. They are called lines of parabolic points.

We notice that the SIS of fast phonons $\left(\Sigma_{0}\right)$ have no lines of parabolic points. When one approaches VPA line (Fig. 3 point no. 2) and PA line (Fig. 3 point no. 7) $\Sigma_{0}$ previously rounded edges becomes sharp, and the Gaussian curvature $\Gamma$ becomes large. Hence, according formulas (14) and (15), phonons with propagation vectors directed toward points lying on these edges are defocused. Inspection of Table 1 shows that the sheets $\Sigma_{1}$ and $\Sigma_{2}$ have sharp edges too.

The behavior of $\Sigma_{1}$ and $\Sigma_{2}$ sheets is more complicated. On the isotropy line $\left(s_{3}=0\right)$ outer and inner sheets coincide and all sheets $\Sigma_{j}(j=0,1,2)$ are perfectly spherical. When one moves along $\sigma_{P}=-1 / 3$-line dimples emerge, and next, they develop into furrows. When one changes $s_{2}$ and $s_{3}$ these dimples evolves - become narrow or broader and within them new structures emerge.

\subsection{Behaviour of polarization of phonons}

In the case of familiar compounds (as a rule placed in the region II of ST) the fast phonons are quasilongitudinal, whereas slow and medium are quasitransverse. However, with the exception of the region II, there exist directions in which fast phonons are quasitransverse whereas slow and medium phonons are quasilongitudinal. In Table 2 we illustrate this unusual situation for the points no. 1 and 7 of $\sigma_{P}=-1 / 3$ line (cf. Fig. 3). Segments of $\Sigma_{0}$ subtending

Table 2. Sheets of slowness surfaces for cubic elastic media for all kinds of LAPs. Various sheets differ by linear dimensions. Polarization anomalies are indicated by grey tones

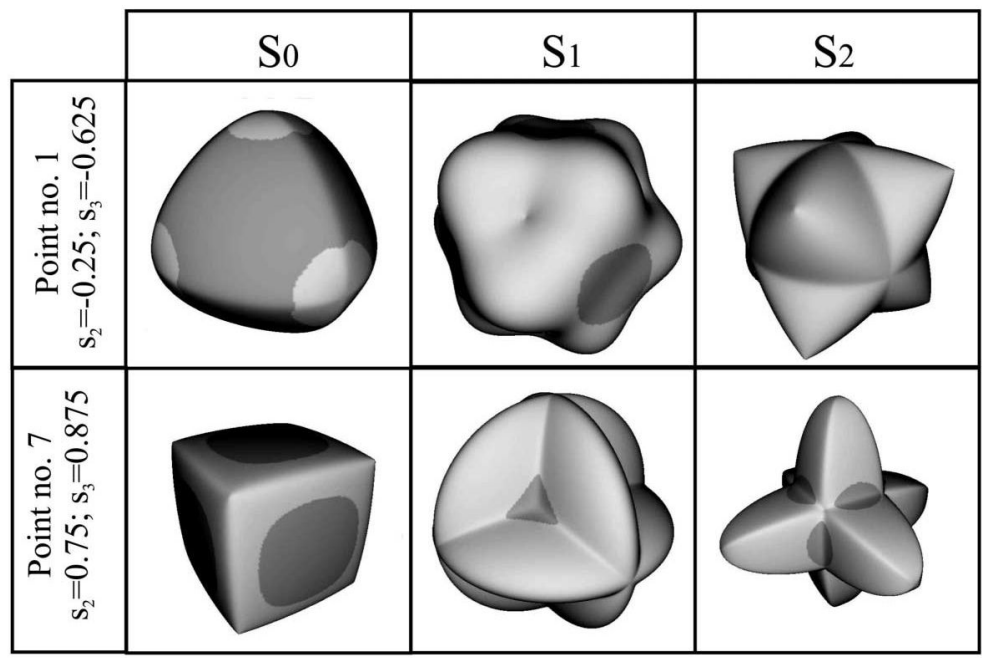


body angles for which phonons are quasitransverse are indicated by white colour. The remaining gray part of $\Sigma_{0}$ corresponds to quasilongitudinal phonons. Analogously segments of $\Sigma_{1}$ and $\Sigma_{2}$ sheets for which slow phonons are quasilongitudinal are grey, and the remaining part corresponding to transverse phonons are white.

\subsection{Energy focusing patterns}

From Eqs. (14), (15) it follows that to parabolic lines there corresponds infinite, but integrable energy density $e$. Lines lying in the chosen plane on which the density of energy transported by phonons is infinite are called caustics. In fact in experiments with ballistic phonon beams mainly caustics are observed. In Table 3 we collected focusing patterns corresponding to slowness surfaces assembled in Table 1. These patterns are obtained with the use of MC AnScat program of Monte Carlo simulations [15]. A bolometer is placed in various points of the plane perpendicular to the z-axis. Regions in which the energy density is large are dark. In white regions $e(\mathbf{r})=0$. White structures corresponds to antifocusing parts of $\Sigma_{j}(j=0$, 1, 2). Dark structures reflect the existence of parabolic lines.

\subsubsection{Polarization enhancement factors}

For each set $\left(\hat{\mathbf{n}}, s_{2}, s_{3}\right)$ three mutually perpendicular polarization vectors $\mathbf{e}\left(\hat{\mathbf{q}}, j ; s_{2}, s_{3}\right)$ $(j=0,1,2)$ specify a Cartesian set of axes $\mathrm{E}$.

Generally orientation of the introduced set of Cartesian axes is now not arbitrary and changes with $\hat{\mathbf{q}}, s_{2}$ and $s_{3}$. The quantitative characterization of a rotation of $\mathrm{E}\left(\hat{\mathbf{q}}, s_{2}, s_{3}\right)$ with changing $\hat{\mathbf{q}}$ and with varying elasticity parameters is a quite complicated task [5].

Consider $j$-th mode for arbitrarily selected values of $s_{2}$ and $s_{3}$. To characterize the rate of change of $\mathrm{E}$ we introduce the enhancement factor $\mathrm{A}_{e}(\hat{Q})$ resembling the familiar enhancement factor for the group velocity (15). Let us choose a bundle of wave vectors around $\hat{\mathbf{q}}$ subtending the solid angle $\Delta \Omega_{q}(\hat{\mathbf{q}})$. To this bundle there corresponds a bundle of polarization vectors around $\mathbf{e}(\hat{Q})$ subtending the body angle $\Delta \Omega_{e}^{(j)}(\hat{\mathbf{q}})$. We may regard $\mathrm{A}_{e}(\hat{Q})$ as the limit of the ratio of solid angles $\Delta \Omega_{q}(\hat{\mathbf{q}})$ and $\Delta \Omega_{e}^{(j)}(\hat{\mathbf{q}})$ when $\Delta \Omega_{q}(\hat{\mathbf{q}}) \rightarrow 0$

$$
\mathrm{A}_{e}^{(j)}(\hat{Q}) \equiv \lim _{\Delta \Omega_{q}(\hat{\mathbf{q}}) \rightarrow 0} \frac{\Delta \Omega_{q}(\hat{\mathbf{q}})}{\Delta \Omega_{e}^{(j)}(\hat{\mathbf{q}})}
$$

Since for each $j$ the field of polarization vectors is not potential $\left(\operatorname{rot}_{q} \mathbf{e}(\hat{Q}) \neq 0\right)$, a closed surface perpendicular to set of polarization vectors $\mathbf{e}(\hat{Q})(\hat{\mathbf{q}} \in 4 \pi)$ does not exist. This means that, differently than the enhancement factors of the group velocity, the polarization enhancement factors $\mathrm{A}_{e}(\hat{Q})$ are not related to local geometrical characteristics of some surfaces (such as, for example, the Gaussian curvature).

Using our program [15] we performed Monte Carlo experiments and obtained maps of $A_{e}(\hat{Q})$ for cubic auxetic media defined by points no. 1-7 on the $\sigma_{P}=-1 / 3-$ line. They are shown in Table 4. 
Table 3. Energy focusing patterns corresponding to SIS gathered in Table 1. Gray tones reflect value of energy density $e(\mathbf{r})$. Regions where $e(\mathbf{r})$ is large are intensively dark. Dark structures are caustics reflecting the presence of lines of parabolic points. White structures corresponds to regions antifocusing parts of $\Sigma_{\mathrm{j}}(j=0,1,2)$ where $\Gamma$ is large

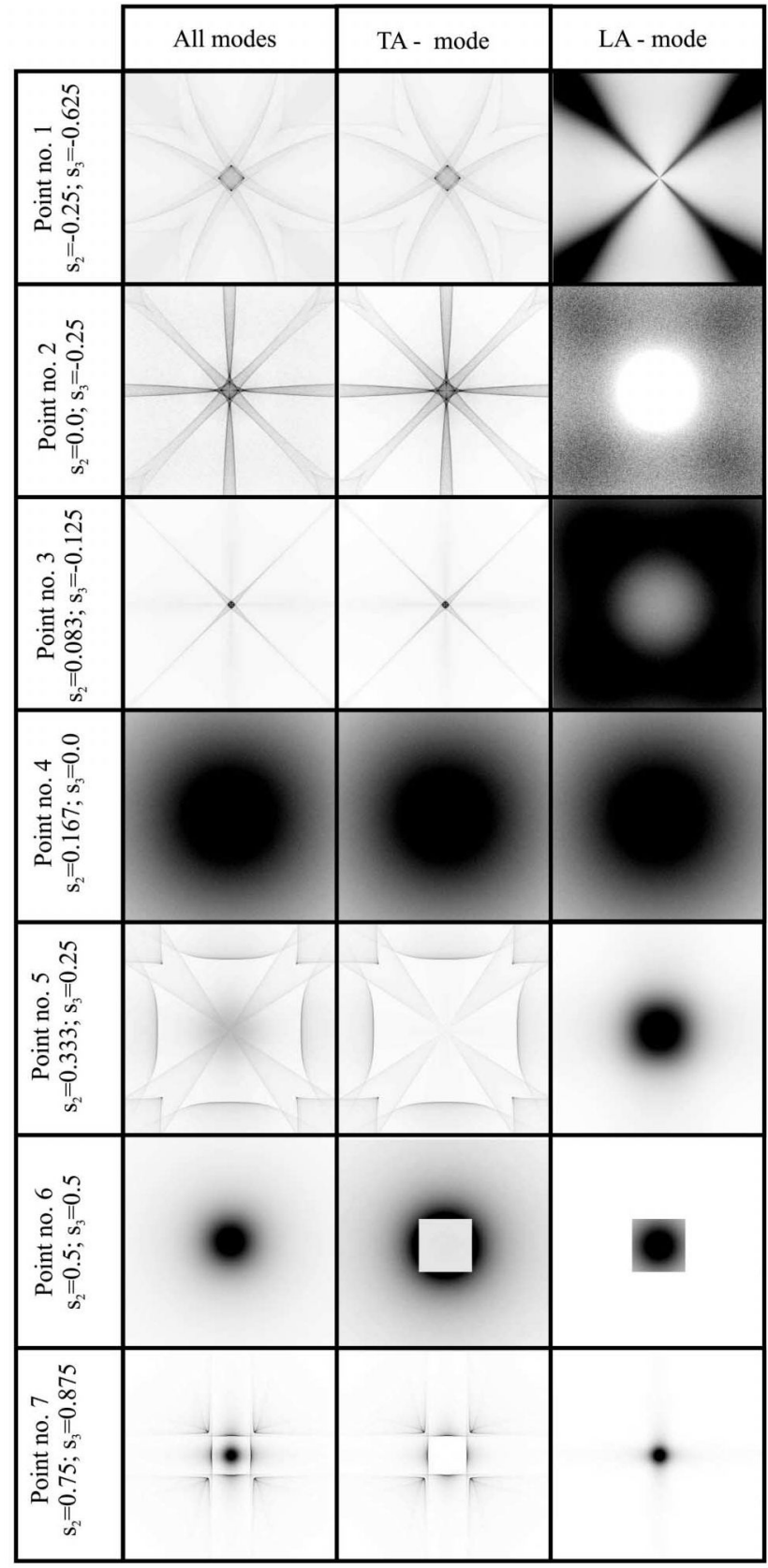


Table 4. The $\left(\theta_{e}, \phi_{e}\right)$ maps of the polarization enhancement factors for all $j$ modes and all points of the $\sigma_{P}=-1 / 3$-line. Dark regions correspond to large enhancement factors. The darkness scale of different patterns is not the same

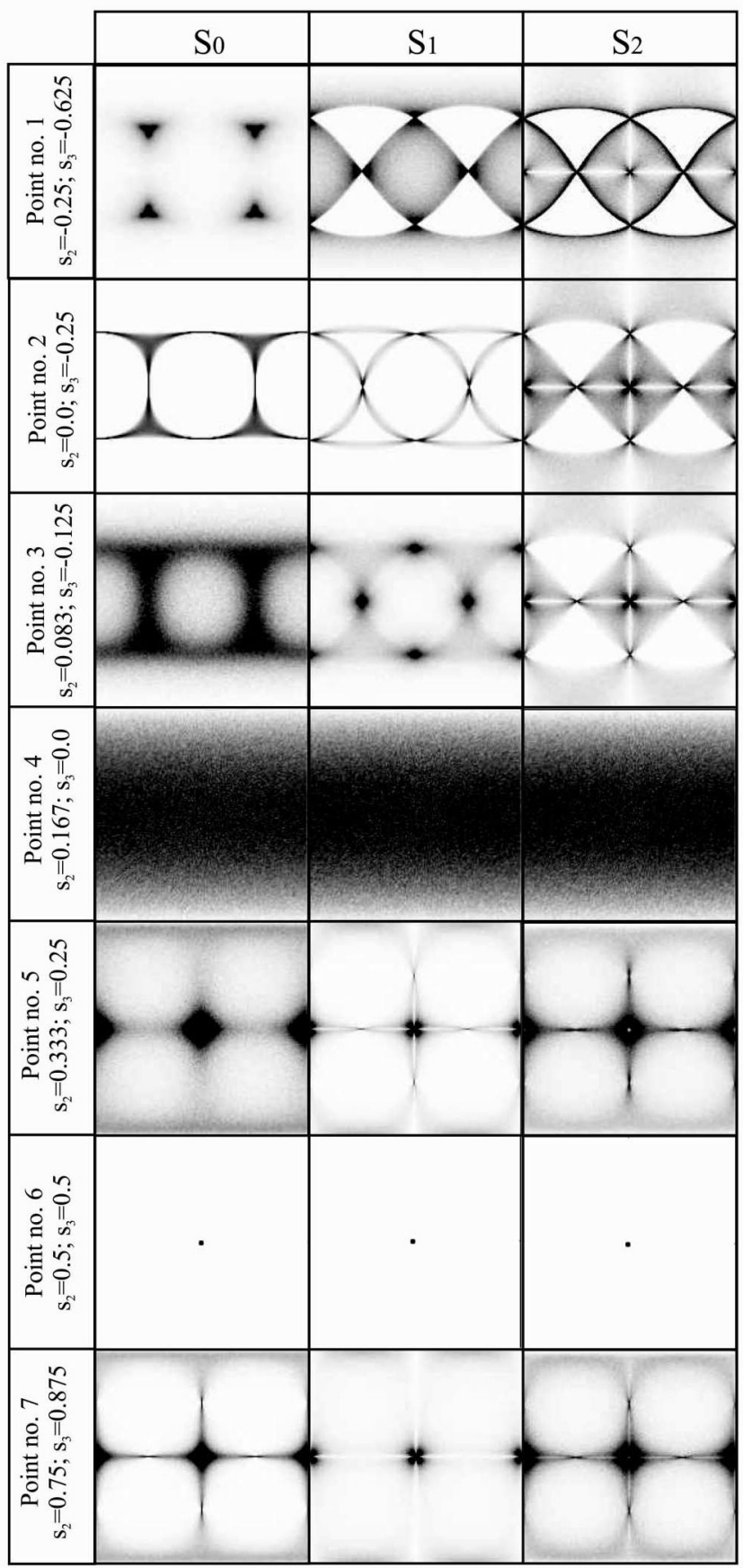


The $<001\rangle$ axes are represented by a points located in the center of the square-shaped region of the polar and azimuthal angles, by the centers of their vertical sides and by whole horizontal sides (cf. patterns belonging to the sixth row of Table 4). Three-leafclovers seen in the first pattern of the first row surround the $\langle 111\rangle$. Spots seen in the second element of the third row are centered around [110] and the equivalent directions (four of them are placed on vertical sides of the square). All "caustic" lines which are seen in patterns from Table 4 join high symmetry directions. In all regions there exist white "districts" where polarization vectors are strongly defocused. When moving toward AAL a very strong focusing of polarization vectors is observed in $\langle 100\rangle$ directions (sixth row of Table 4). This is in agreement with observation that on AAL these vectors are fixed along fourfold symmetry axes. One should underline that intensities of various patterns depicted in Table 4 differ immensely.

\section{References}

[1] R. H. Baughman, Auxetic materials: Avoiding the shrink, Nature 425, 667-670 (2003).

[2] R. S. Lakes and R. Witt, Making and Characterizing Negative Poisson's Ratio Materials, International Journal of Mechanical Engineering Education 30, 50 (2002).

[3] K. E. Evans and A. Alderson, Auxetic materials: functional materials and structures from lateral thinking, in: Advanced Materials (Weinheim, Germany) 12, 617-628 (2000).

[4] R. H. Baughman, J. M. Shacklette, A. A. Zakhidov, and S. Stafström, Negative Poisson's ratios as a common feature of cubic metals, Nature 392, 362-365 (1998).

[5] T. Paszkiewicz and M. Pruchnik, Acoustic phonons in cubic media: properies of their polarizations and the diffusion coefficient, Eur. Phys. J. B 24, 91-99 (2001).

[6] T. Paszkiewicz, M. Pruchnik, and P. Zieliński, Unified description of elastic and acoustic properties of cubic media: elastic instabilities, phase transitions and soft modes, Eur. Phys. J. B 24, 327-338 (2001).

[7] T. Paszkiewicz, M. Pruchnik, and S. Wolski, Anisotropy of elastic characteristics of cubic media, in preparation.

[8] J. P. Wolfe, Imaging Phonons. Acoustic Wave Propagation in Solids, Cambridge University Press, Cambridge, 1998.

[9] T. Paszkiewicz and M. Pruchnik, Kinetic description of the phonon-pulse propagation and phonon images of crystalline solids, Physica 232, 747-768 (1996).

[10] D. Arbruster and G. Dangelmayr, Topological singularities, Z. Phys. B - Condensed Matter, 52, 87-94 (1983).

[11] A. G. Every, General closed-form expressions for acoustic waves in elastically anisotropic solids, Phys. Rev. B 22, 1746-1760 (1980).

[12] T. Paszkiewicz and M. Wilczyński, Scattering of long-wavelength acoustic phonons by isotopic impurities. Spectra of the collision integral and diffusion equation for crystalline media with cubic symmetry, Z. Phys. B 88, 5-15 (1992).

[13] T. Paszkiewicz and M. Wilczyński, Influence of isotopic and substitutional atoms on the propagation ofphonons in anisotropic media, in: G. K. Horton, A. A. Maradudin (eds.), Dynamical Properties of Solids vol. 7, Phonon Physics, the Cutting Edge, North Holland, Amsterdam, pp. 257-348, 1995.

[14] U Schärer and P. Wachter, Negative elastic constants in intermediate valent Sm La S, Solid State Commun. 96, 497-500 (1995).

[15] W. M. Gancza, I. A. Obukhov, T. Paszkiewicz, and B. A. Danilchenko, Experiments on propagation and elastic scattering of down-converting beams ofphonons, Comp. Meth. Sci. Techn. 7, 7-46 (2001). 